\title{
Publish or Perish? Neue Ansätze in der Evaluation von Wissenschaftlerleistungen
}

\author{
Dana Mietzner*, Frank Hartmann
}

\section{Zusammenfassung}

Wenn es um die Bewertung von wissenschaftlichen Leistungen geht, berücksichtigt die aktuelle Evaluationspraxis nur eine begrenzte Anzahl an Dimensionen von Qualität und Relevanz von Forschung und Lehre und entspricht damit nicht mehr den Anforderungen, die aus der Komplexität des Wissenschaftssystems resultieren. Mit dem im Rahmen dieses Beitrages skizzierten ACUMEN-Portfolio wird ein ganzheitlicher Bewertungsansatz prototypisch vorgestellt, der Differenzierungen im Hinblick auf unterschiedliche Wissenschaftsdisziplinen ermöglicht und die Perspektive des einzelnen Wissenschaftlers in den Mittelpunkt der Evaluation rückt. Das ACUMEN-Portfolio ist ein Ergebnis des im Europäischen 7. Forschungsrahmenprogramm durchgeführten Projektes ACUMEN - Academic Careers Understood trough Measurements and Norms. Das Projekt wurde von März 2011 bis Februar 2014 gemeinsam von neun Partnern' ${ }^{1}$ aus sieben Ländern unter der Leitung der Universität Leiden (NL), Centre for Science and Technology Studies (CWTS) durchgeführt.

\section{Abstract}

When researchers are evaluated, current evaluation practices consider only a limited number of aspects in terms of quality and relevance for research and teaching. However, today, the scientific system is characterised by increasing levels of complexity and dimension that are not fully represented by predominant evaluation practices and their methodological shortcomings. In this article, we present the ACUMEN portfolio prototype as a holistic approach, which considers differences between several scientific disciplines and puts the perspective of the single researcher in the focus of evaluation. The ACUMEN portfolio is one result of the European FP 7 research project ACUMEN Academic Careers Under-stood trough Measurements and Norms. The project took place from March 2011 until February 2014 in joint collaboration with nine partners from seven countries under the lead of the University of Leiden (NL), Centre for Science and Technology Studies (CWTS).

\section{AUSGANGSSITUATION}

Wenn es um die Bewertung von wissenschaftlichen Leistungen, z. B. im Zusammenhang mit der Bewerbung um eine Professur, eine Position als Forschungsgruppenleiter/in, einen akademischen Preis, die Aufnahme in ein Doktorandenprogramm oder eine individuelle Karrierebewertung geht, ist die Evaluationspraxis heute durch bibliometrische Messkriterien wie den Journal Impact-Faktor (IF) oder die Anzahl der Publikationen in internationalen peer-reviewed Journals geprägt. Die Anzahl der Publikationen in hochrangigen Journals mit einem entsprechenden Impact
Faktor haben - auch in Abhängigkeit von der jeweiligen wissenschaftlichen Disziplin - durchaus ihre Berechtigung. Dennoch weist die ausschließliche Berücksichtigung dieser Faktoren Limitierungen auf wenn es darum geht, Wissenschaftler unter Beachtung unterschiedlicher und vielfältiger Karriere- und Lebenspfade und unter Berücksichtigung des jeweiligen Kontextes und Ziels der Evaluation differenziert zu betrachten (Wildgaard 2014, Tatum \& Wouters 2013, Bar Ilan et al. 2014).

Im ACUMEN-Projekt gingen die beteiligten Projektpartner von der grundlegenden Annahme aus, dass sich in den vergangenen Jahren europaweit
Diskrepanzen zwischen den externen Funktionen von Wissenschaft und Forschung und den internen Bewertungskriterien für die Leistungsfähigkeit und Qualität wissenschaftlichen Arbeitens und Forschens herausgebildet haben. Angesichts zunehmender Differenziertheit der Funktionen von Wissenschaft und Forschung, etwa hinsichtlich der ökonomischen Verwertbarkeit, der Anwendungsvielfalt auch in solchen Bereichen wie Soziales und Kultur oder der unterschiedlichsten Beratungsmöglichkeiten von Wissenschaft für Politik, Wirtschaft, Verwaltung oder auch NGOs, zeigen sich die Grenzen interner Bewertungslogiken besonders

\footnotetext{
1 Leiden University (Niederlande), Bar Ilan University (Ramat Gan, Israel), Agencia estatal consejo superior de investigaciones cientificas (Madrid, Spanien), University of Wolverhampton (Großbritannien), Estonian Research Council (Tartu, Estland), Humboldt-Universität (Berlin, Deutschland), Technische Hochschule Wildau (Wildau, Deutschland), Danmarks Biblioteksskole (Kopenhagen, Dänemark), eHumanities Group - KNAW (Amsterdam, Niederlande).
} 
deutlich. Dies äußert sich beispielsweise:

- in der Berücksichtigung einer nur begrenzten Anzahl an Dimensionen von Qualität und Relevanz von Forschung und Lehre, oft vor dem Hintergrund monodisziplinärer Perspektiven,

- in einem wachsenden Druck auf traditionelle Formen der Evaluation durch die zunehmende Größe und Komplexität des Wissenschaftssystems,

- in neuen Anforderungen an das Evaluationssystem aufgrund der Veränderungen in der Art und Weise der Produktion wissenschaftlichen Wissens und dessen Kommunikation,

- in der Erkenntnis profunder methodologischer Begrenzungen der Aussagefähigkeit von Zitationsmethoden sowie

- im Gender bias bei der Bewertung der Leistungsfähigkeit von Wissenschaftlern.

Für die Lösung dieser Probleme wollte auch das ACUMEN-Projekt einen Beitrag leisten, der im ACUMEN-Portfolio als einem neuen Bewertungsinstrument zum Ausdruck kommt. Bei der Entwicklung eines neuen Bewertungsansatzes waren (1) Differenzierungen bezüglich unterschiedlicher Disziplinen, unterschiedlicher Typen von Forschungseinrichtungen sowie staatlicher Evaluationspraktiken zu berücksichtigen. (2) Die Perspektive des einzelnen Wissenschaftlers sollte in den Mittelpunkt der Evaluation rücken. Diese selbst sollte weniger als Outputbewertung verstanden werden, sondern vielmehr als ein Kommunikationsprozess zwischen Wissenschaftler und Evaluator, in welchem die Wirkungen der Evaluation auf den Wissenschaftler selbst sowie auf den Evaluator eruiert und bewertet werden. (3) Generelle Evaluationskonzepte, die sich hinter Begriffen wie "Excellenz", "Originalität" oder "Soziale Relevanz" verbergen, sollten aufgebrochen werden, um die soziale und kulturelle Vielfalt unterschiedlicher Evaluationspraktiken besser verstehen zu können.

\section{ENTWICKLUNG DES ACUMEN- PORTFOLIOS ALS NEUER ANSATZ IN DER EVALUATIONSPRAXIS - METHODISCHER ANSATZ}

Um geeignete Faktoren auswählen zu können, die im Rahmen des ACUMEN-Portfolios Berücksichtigung finden sollten, wurden in einem ersten Schritt unterschiedliche Typen an neuen und ergänzenden Indikatoren identifiziert und im Hinblick auf ihre Tauglichkeit für die Evaluation von Wissenschaftlerleistungen durch die am Projekt beteiligten Experten bewertet. Es wurden web Impactmetrics-Indikatoren, die auf online verfügbaren Präsentationen, digitaIen Büchern, Syllabi, online verfügbaren Videos oder Wissenschaftsblogs basieren, herangezogen und Möglichkeiten der automatisierten Suche dieser Indikatoren untersucht. Auch die in digitalen Bibliotheken, wie z. B. CiteSeer, Google Scholar und Google Books, verfügbaren Informationen wurden vor dem Hintergrund der Suche nach Indikatoren bewertet (Kousha \& Thelwall 2014a). Dabei wurde z.B. auch die Anzahl der Downloads als ein Indikator für den Nutzen und die Verbreitung von wissenschaftlichen Erkenntnissen berücksichtigt (Kousha \& Thelwall 2014b). Es wurde auch hinterfragt, welche Rolle Google Scholar als Zitationsmaßstab durch die Messung der Anzahl an Zitationen oder die Ermittlung des h-index ${ }^{2}$ spielen kann (Hirsch 2005, Bar Ilan 2008). Im Ergebnis dieser Untersuchung kann festgestellt werden, dass eine Vielzahl an möglichen webbasierten Indikatoren existiert, diese jedoch aufgrund fehlender Qualitätskontrolle sorgfältig ausgewählt und verwendet werden müssen und somit nur eine Ergänzung zu herkömmlichen Indikatoren darstellen können.
Zusätzlich wurden sogenannte Altmetrics-Indikatoren („alternative Metrics" oder "Article Level Metrics") als neue Form der Impact-Messung diskutiert, die den Einfluss eines Autors auf der Ebene eines einzelnen Artikels oder Buches auf das jeweilige Forschungsfeld aufzeigen und bewerten helfen. Typische Altmetrics-Indikatoren erfassen die Anzahl von Aufrufen (sogenannte Views), Downloads, Lesern/Abonnenten oder Kommentaren auf Mendeley (Referenz Manager), YouTube (Kousha \& Thelwall 2012, Kousha et al. 2012), Academia. edu (vgl. Thelwall \& Kousha 2014a) oder researchgate.net (vgl. Thelwall \& Kousha 2014b) sowie Blog- oder Tweet-Zitationen. Damit adressieren diese Faktoren eher die Reichweite in einer breiteren Öffentlichkeit als in der jeweiligen Wissenschaftsdisziplin. Diese Form von Indikatoren kann insbesondere dann eine Rolle spielen, wenn herkömmliche Indikatoren nicht verfügbar oder für den spezifischen Evaluationszweck als nicht geeignet angesehen werden (ACUMEN 2014a: 17 f.).

Neben der Betrachtung der webbasierten Indikatoren wurden im Rahmen des ACUMEN-Projektes Internetpräsentationen von 1.500 viel zitierten europäischen Wissenschaftlern ausgewertet, um Rückschlüsse auf den Nutzen von Informationen von Internetpräsentationen für ausgewählte Inhalte des Portfolios ziehen zu können (vgl. Kousha \& Thelwall 2013). Insgesamt konnte festgestellt werden, dass zwar immerhin über $70 \%$ Wissenschaftler über eine Internetpräsenz verfügen, das Potenzial aber noch nicht ausgeschöpft ist und somit die Möglichkeiten des Wissensaustauschs und der Wissensverbreitung noch nicht in vollem Umfang genutzt werden (vgl. Más-Bleda \& Aguillo 2013).

In einem nächsten Schritt wurde der Portfolio-Ansatz in einem iterativen Vorgehen im Rahmen von zwei Expertenworkshops und mehreren Feedbackrunden schrittweise entwi-

${ }^{2}$ h-index = Hirsch-Index, gemessen wird die Anzahl der Zitierungen eines Wissenschaftlers verteilt auf alle seine Publikationen, $h$-index = persönlicher "Impact-Faktor" im jeweiligen Wissenschaftsgebiet. Zur Berechnung des h-index kann das Web of Science (WoS) genutzt werden (kostenpflichtig). Dabei muss jedoch beachtet werden, dass nur die im WoS erfassten Publikationen berücksichtigt werden. Google bietet inzwischen auf Basis von Google Scholar kostenlose Zitationsanalysen an. Siehe: http://scholar.google.com/citations. 


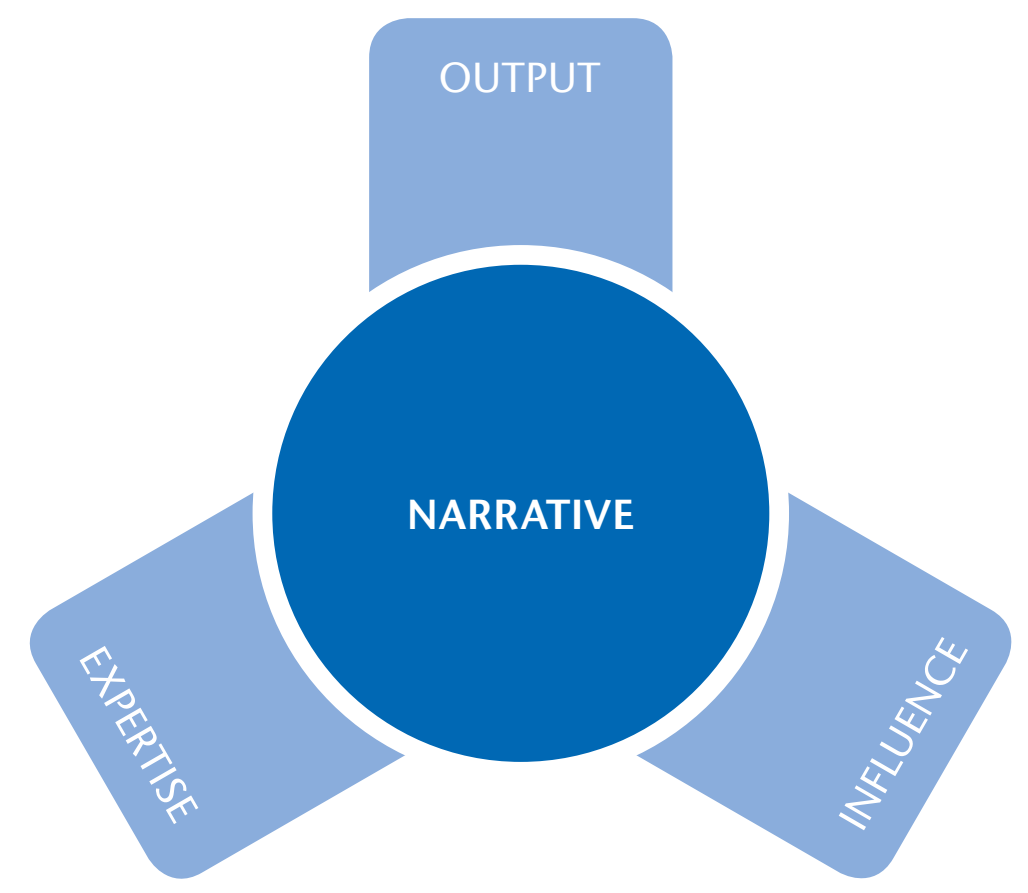

Abb. 1) ACUMEN-Portfolio und assoziierte Sub-Portfolios.

ckelt, getestet, überarbeitet sowie mit der angesprochenen Zielgruppe getestet und potenziellen Evaluatoren diskutiert. In diesen Prozess waren die Autoren intensiv einbezogen. Die im Rahmen der Expertenworkshops (Januar 2013 in Madrid und März 2013 in Kopenhagen) einbezogenen Experten aus sieben Ländern sind ausgewiesen im Bereich Scientometric und verwandten Wissenschaftsbereichen. Somit konnte auf eine breite Expertenbasis zu bibliometrischen Faktoren, web impact metrics/web indicators und Altmetrics-Indikatoren zurückgegriffen werden. Im Rahmen der Expertenworkshops wurden verschiedene Teams gebildet und schrittweise Indikatoren gesammelt, bewertet, überprüft, hinterfragt und konsensorientiert in das ACUMEN-Portfolio überführt. Die vorgeschlagenen Indikatoren wurden mithilfe von Bewertungsmethoden systematisch zusammengefasst und zunehmend fokussiert. Durch das schrittweise Vorgehen und den Wechsel von intensiven Workshop-Phasen, Nachbereitungen und virtuellen Abstimmungen und Diskussionen konnten schrittweise Redundanzen reduziert und ein ganzheitlicher Portfolioansatz entwickelt werden.

Das zunächst prototypisch entwickelte Portfolio wurde anhand von typischen Anwendungsfällen weiter erprobt und im Hinblick auf seine Tauglichkeit in unterschiedlichen Evaluationsszenarien untersucht. In diesem iterativen Prozess wurde deutlich, dass eine ausschließliche Verwendung von outputorientierten Indikatoren nur begrenzt geeignet ist, um die Komplexität im Wissenschaftsumfeld und die Vielfalt der Erfahrungen, Expertise, Einflüsse und individuellen, persönlichen Kompetenzen von Wissenschaftlern in unterschiedlichen Phasen ihrer Karriereentwicklung und Evaluationsszenarien abzubilden.

\section{ACUMEN-PORTFOLIO - EIGENSCHAFTEN}

Ziel des ACUMEN-Portfolios ist es, einen ganzheitlichen Überblick zu der jeweiligen Expertise, zum generierten Output und dem Einfluss (Influence) des Wissenschaftlers darzustellen. Zudem sollen die Wissenschaftler die Möglichkeit haben, besondere Fähigkeiten, Ergebnisse oder Ereignisse hervorzuheben und den Arbeitskontext deutlich zu machen. Das ACUMEN-Portfolio wird zu diesem Zweck in die drei Bereiche Expertise, Output und Influence aufgegliedert (vgl. Abbildung 1). Die jeweiligen Bereiche des Portfolios werden mit einem Set an qualitativen und quantitativen Indikatoren und Referenzen hinterlegt. Zudem wird das Portfolio mit einem Bericht un- terlegt, der die drei Portfoliobereiche miteinander verbindet und dem Wissenschaftler die Möglichkeit bietet, auf die jeweilige Evaluationssituation bezogen, qualitative Informationen an den Evaluator zu übermitteln und besonders relevante Aspekte in „erzählerischer Form“ hervorzuheben. Mit diesem Instrument wird das Portfolio dem Anspruch gerecht, die Kommunikation zwischen Wissenschaftlern und Evaluatoren anzuregen.

In den nachfolgenden Darstellungen sind die Indikatoren der jeweiligen Sub-Portfolios im Überblick dargestellt und kurz erläutert (ACUMEN 2014b: 101 ff.). Dabei werden die einzelnen Sub-Portfolios in einzelne Indikatorenkategorien untergliedert, die wiederum durch einzelne Faktoren näher beschrieben werden. 
Tab. 1: Acumen-Sub-Portfolio-Expertise

\begin{tabular}{l|l|l} 
Expertise & Sub-Faktor & Beschreibung und Nachvollziehbarkeit
\end{tabular}

- Fassen Sie Ihre theoretische Expertise in wenigen Sätzen zusammen Wissenschaftliche Theorie und belegen Sie diese (z. B. mit einem zitierten Artikel). Diese Expertise Expertise kann ein theoretisches Konzept oder ein Paradigma sein, dem Sie folgen. Wenn dies nicht auf Sie zutrifft, z.B. weil sie empirisch arbeiten, schreiben Sie bitte: „Trifft nicht auf mein Forschungsgebiet zu.“

Gegenstand

- Fassen Sie Ihre gegenstandsspezifische Expertise in wenigen Sätzen zusammen und belegen Sie diese (z. B. mit einem zitierten Artikel). Das betrifft normalerweise Ihre Hauptforschungsgebiete.

Methode

- Fassen Sie Ihre methodologische Expertise in wenigen Sätzen zusammen und belegen diese (z.B. mit einem zitierten Artikel). Dies bezieht sich auf die wichtigsten Methoden, die Sie im Forschungsprozess verwenden.

Originalität

- Fassen Sie die Originalität Ihrer Forschungsarbeit in einigen wenigen Sätzen zusammen und belegen Sie diese.

\section{Wissenstransfer- Überprüfung/ \\ Expertise Reviewing}

Entrepreneurship

Ausbildungs- Kurse gelehrt/

Expertise

entwickelt

Weitere

Technologische

Expertise

Methoden

Tools + Labor-

ausstattung

Software

Datenmanagement,

Daten

Kommunikations-

Expertise

Sprachen

Präsentationen

Veröffentlichungen

Öffentliches

Engagement

\begin{tabular}{|l|l|}
\hline $\begin{array}{l}\text { Organisations- } \\
\text { Expertise }\end{array}$ & Management \\
\hline & Beratung \\
\hline Projektleitung \\
\hline
\end{tabular}

Kooperation

Administration und Ausschüsse

Weitere Expertise
- Geben Sie die Anzahl von (a) Konferenzbeiträgen und (b) Zeitschriftenartikeln an, die Sie begutachtet haben, und (c) geben Sie Beispiele für entsprechende Journals oder Konferenzen an (Benennen Sie dabei die Top 3.).

- Führen Sie Ihre Entrepreneurship-Aktivitäten auf (z. B. Initiierung oder Beteiligung an Spin-Offs, gemeinsame Projekte mit der Industrie (Benennen Sie dabei die Top 3.).

- (a) Geben Sie die etwaige Anzahl von Lehrstunden an

- (b) Beschreiben Sie Ihre Lehrveranstaltungen (ohne online-Lehrveranstaltungen, diese werden unter Output erwähnt). (Benennen Sie dabei die Top 3.).

- Geben Sie beispielsweise Summer Schools oder Exkursionen an.

- Fassen Sie Ihre Methodenexpertise in wenigen Sätzen zusammen und belegen Sie diese.

- Charakterisieren Sie Ihre Laborausstattung und spezielle Methoden, die Sie einsetzen. Belegen Sie dies z.B. mit Projekten oder zitierten Publikationen.

- Beschreiben Sie kurz Ihre Software-Expertise und belegen Sie diese.

- Beschreiben Sie kurz Ihre Datenmanagement-Expertise. Welche Datenbasen nutzen Sie? Belegen Sie diese Expertise.

- Listen Sie Ihre Fremdsprachenkenntnisse auf, einschließlich des Sprachniveaus.

- Geben Sie Ihre Keynotes/Invited Talks für Institutionen oder Konferenzen an (Benennen Sie die Top 3.).

- Geben Sie Ihre Auszeichnungen für Publikationen an (Benennen Sie die Top 3.).

- Geben Sie Beispiele für Interviews in Rundfunk- und Fernsehmedien (Benennen Sie die Top 3.).

- Beschreiben Sie wahrgenommenen Managementfunktionen (Benennen Sie die Top 3.).

- Nennen Sie beratene Institutionen (Benennen Sie die Top 3.).

- Nennen Sie Projekte und geleitete Teams, einschließlich Größe der Teams und ob es sich um internationale Kooperationen handelt (Benennen Sie die Top 3.).

- Mitarbeit in Projekten und Teams, einschließlich Größe des Teams und ob es sich um internationale Kooperationen handelt. Beschreiben Sie Ihre Position im Team (Benennen Sie die Top 3.).

- Beschreiben Sie Ihre administrativen Funktionen, einschließlich Mitgliedschaften in Ausschüssen, Vorsitze oder Sekretärsaufgaben, Arbeiten als Organisator von Konferenzen ... (Benennen Sie die Top 3.).

Nennen Sie weitere Expertisen und belegen Sie diese (Benennen Sie dabei die Top 3.). 
Tab. 2: ACUMEN-Sub-Portfolio-Output

\section{Expertise}

Wissenschaft

Bücher

Buchkapitel

Reviews

Leitartikel/ Editorials

Zeitschriftenartikel

Konferenzbeiträge

Kommunikation

mit der

Öffentlichkeit

Lehre

Bücher

Online-Kurse

Betreute Studenten

Akademische Kommunikation im Internet und in Social Media

Online-Präsenz

Online-Beiträge

Datensätze, Software, Tools, Instrumente

\section{Beschreibung und Nachvollziehbarkeit}

- Anzahl von Ihnen publizierter wissenschaftlicher Bücher (ausgenommen Selbstverlag):

- Liste der von Ihnen publizierten Bücher (Benennen Sie die Top 3.).

- Anzahl der von Ihnen publizierten Buchkapitel:

- Liste der von Ihnen publizierten Buchkapitel (Benennen Sie dabei die Top 3.).

- Anzahl der von Ihnen publizierten Buchreviews:

- Anzahl der von Ihnen publizierten Leitartikel/Editorials:

- Anzahl der von Ihnen begutachteten Zeitschriftenartikel oder Konferenzbeiträge:

- Liste der begutachteten Zeitschriftenartikel oder Konferenzbeiträge (Benennen Sie die Top 3.).

- Anzahl der von Ihnen publizierten Tagungsbeiträge (Conference Abstracts), Podiumsdiskussionen oder Poster:

(Berücksichtigen Sie nicht-publizierte Konferenzbeiträge nicht.)

- Anzahl der von Ihnen publizierten Zeitungsartikel oder Magazinbeiträge:

- Liste der von Ihnen publizierten Zeitungsartikel oder Magazinbeiträge (Benennen Sie die Top 3.).

- Anzahl der von Ihnen publizierten Beiträge (ausgenommen Wikipedia oder vergleichbare Medien):

- Liste der von Ihnen publizierten Beiträge (Benennen Sie die Top 3.).

- Anzahl der von Ihnen publizierten Bücher oder Artikel:

- Liste der von Ihnen publizierten Bücher oder Artikel (Benennen Sie hierbei die Top 3.).

- Anzahl der von Ihnen veröffentlichten Textbücher (ausgenommen Selbstverlag):

- Liste der von Ihnen publizierten Textbücher (Benennen Sie hier ausschließlich die Top 3.).

- Liste der von Ihnen entwickelten Kurse (einschließlich MOOCs), wann entwickelt? Art der Unterlagen und Anzahl an Studenten jährlich (Benennen Sie die Top 3.).

- Hauptbetreuer von noch nicht graduierten Studenten: Studenten

- Hauptbetreuer von graduierten Masterstudenten: Studenten

- Hauptbetreuer von Doktoranden: Doktoranden

- Listen Sie Ihre Online-Präsenz auf: Accounts in Social Media, genutzt für akademische Zwecke, Academic Network Accounts, Digital Repository Accounts, Websites. Wenn zutreffend, geben Sie an, wie aktiv Sie bezogen auf eine Seite sind (Posts pro Jahr bzw. Monat etc., Twitter, Blogs, ResearchGate, SlideShare) (Benennen Sie die Top 3.).

- Geben Sie Beispiele für andere Online-Beiträge in der wissenschaftlichen Diskussion an (Benennen Sie die Top 3.).

- Anzahl publizierter Datensätze:

- Kurze Beschreibung der publizierten Datensätze (Benennen Sie hierbei nur die Top 3.).

Anzahl der entwickelten Software, Tools und Instrumente: Kurze Beschreibung der entwickelten Software, Tools und Instrumente (Benennen Sie die Top 3.).
Software, Tools, Instrumente 


\section{Expertise}

Schutzrechte

Patente

Entdeckungen

\section{Finanzierung \& \\ Grants}

Finanzierung

Sub-Faktor

Andere
Beschreibung und Nachvollziehbarkeit

- Anzahl der von Ihnen publizierten Patente, Standards, Guidelines:

- Kurze Beschreibung der von Ihnen publizierten Patente, Standards, Guidelines (Benennen Sie die Top 3.).

- Anzahl Ihrer Entdeckungen (z.B. DNA-Sequenzen, Tierarten ...):

- Kurze Beschreibung Ihrer Entdeckungen (Beschreiben Sie hierbei nur die Top 3):

- Anzahl finanzierter Projekte:

- Gesamte Drittmittelfinanzierung:

- Kurze Beschreibung der Drittmittelprojekte (Benennung der Top 3.).

- Beschreiben Sie weitere Arten von Output (Benennung der Top 3.).

Tab. 3: ACUMEN-Sub-Portfolio-Einfluss

\section{Expertise}

Wissenschaft

\section{Sub-Faktor}

Publikationen

Artikel
Beschreibung und Nachvollziehbarkeit

- Anzahl Zitationen für alle Publikationen, Google Scholar:

und durchschnittliche Zahl Zitationen je

Publikation:

- Anzahl Zitationen für alle Publikationen, Web of Science oder Scopus: und durchschnittliche Zahl Zitationen je Publikation:

Anzahl Zitationen für einen Ihrer Top 3 Artikel, Google Scholar: und Scopus oder Web of Science:

Titel:

Publikationsjahr:

Autoren (in Reihenfolge):

Anzahl Zitationen für einen anderen Ihrer Top 3-Artikel, Google Scholar: und Scopus oder Web of Science: Titel:

Publikationsjahr:

Anzahl Zitationen für einen anderen Ihrer Top 3-Artikel, Google Scholar: und Scopus or Web of Science:

Titel:

Publikationsjahr:

Autoren (in Reihenfolge):

h-index, wie in Google Scholar angegeben:

h-index, wie in Web of Science der Scopus hinterlegt: 
Bücher

Alterskorrigierter h-index

Multi-Autorenschaft

Wissenschaftspreise

Herausgeberschaft und Reviewing

ProgrammKomiteearbeit
Zitationen für eines Ihrer Top

3-Bücher, Google Books:

Titel:

Erscheinungsjahr:

Autoren (in Reihenfolge):

Zitationen für ein anderes Ihrer

Top 3-Bücher, Google Books:

Titel:

Erscheinungsjahr:

Autoren (in Reihenfolge):

Zitationen für ein anderes Ihrer Top 3-Bücher, Google Books:

Titel:

Erscheinungsjahr:

Autoren (in Reihenfolge):

[Um Google-Books-Zitationen für ein Buch zu finden, suchen Sie in Google Books nach dem Titel und scannen Sie manuell die Ergebnisse für Zitationen.]

m-Quotient für Google Scholar:

m-Quotient für Web of Science oder Scopus:

[In diesem Portfolio ist der m-Quotient der h-index dividiert durch das akademische Alter.]

Um Multi-Autorenschaft auszugleichen, benutzen Sie entweder (a) oder (b).

a) Durchschnittliche Anzahl von Autoren in Ihren Publikationen, gelistet in Google Scholar (einschließlich Ihrer Person):

b) Anteil von Publikationen, gelistet in Google Scholar, in denen Sie Erstautor sind:

Wenn für Sie Web-of-Science-Publikationen oder Scopus-Publikationen wichtiger sind als Google-Scholar-Publikationen, dann können Sie auf diese verweisen. Bitte machen Sie dies explizit deutlich.

Wissenschaftliche Preise und Ehrungen (regional, national und international) (Benennen Sie die Top 3.).

Ihre Hauptaufgaben als Gutachter, Herausgeber oder redaktioneller Beirat (Editorial Board Member). (Benennen Sie hierzu die Top 3.).

Ihre Mitgliedschaften in Konferenz/Programm-Ausschüssen (Benennen Sie die Top 3.). 
Anzahl der Anhänger (Follower), sofern erheblich, bezüglich Ihrer WebPräsenz (e.g., Academia, Blogs, Twitter) (Benennen Sie die Top 3.).

Online-Diskussionen Social Web Follower

Downloads

Mendeley Leser

Keynotes-

Einladungen

Einfluss auf Gesellschaft
Name der

Social Website

Name der

Social Website

Name der

Social Website

Anzahl der
Anhänger
(Follower):
Anzahl der
Anhänger
(Follower):
Anzahl der
Anhänger
(Follower):

Benennen Sie auch drei interessante Online-Erwähnungen Ihrer Arbeit, die bisher noch keine Erwähnung in diesem Portfolio gefunden haben:

$\begin{array}{ll}\text { Artikelbe- } & \text { Anzahl der } \\ \text { zeichnung } & \text { Downloads } \\ \text { Artikelbe- } & \text { Anzahl der } \\ \text { zeichnung } & \text { Downloads } \\ \text { Artikelbe- } & \text { Anzahl der } \\ \text { zeichnung } & \text { Downloads }\end{array}$

(Top 3 der Downloads)

[Downloads werden manchmal auf den Verlagswebseiten ausgewiesen. Schreiben Sie N/A in das dafür vorgesehene Feld, wenn keine Informationen zu dieser Abfrage vorliegen. Sie können an dieser Stelle auch Downloads für elektronische Berichte oder andere Quellen angeben.]

$\begin{array}{ll}\text { Artikelbe- } & \text { Anzahl der } \\ \text { zeichnung } & \text { Mendeley-Leser } \\ \text { Artikelbe- } & \text { Anzahl der } \\ \text { zeichnung } & \text { Mendeley-Leser } \\ \text { Artikelbe- } & \text { Anzahl der } \\ \text { zeichnung } & \text { Mendeley-Leser }\end{array}$

Um die Anzahl der Mendeley-Leser zu bestimmen, besuchen Sie die Seite "Mendeley.com“ und suchen Sie nach jeder Ihrer Publikationen und entnehmen Sie dem Eintrag die entsprechende Leseranzahl. (Benennen Sie hierbei ausschließlich die Top 3.).

Anzahl Einladungen für Keynote-Talks auf Konferenzen außerhalb Ihres Landes: Anzahl Einladungen für Keynote-Talks auf Konferenzen innerhalb Ihres Landes: Anzahl Einladungen für Keynote-Talks von Universitäten außerhalb Ihres Landes: Anzahl Einladungen für Keynote-Talks von Universitäten innerhalb Ihres Landes:

Liste der Einladungen für alle Arten [einschließlich Name und Ort] (Benennen Sie die Top 3.).

Anzahl publizierter Magazin- oder Zeitungsartikel (geschrieben über Ihre Forschung, nicht von Ihnen)

Beispiele publizierter Magazin- oder Zeitungsartikel (geschrieben über Ihre Forschung, nicht von Ihnen) (Benennen Sie die Top 3):

Beispiele für Websites (über Ihre Forschung, nicht von Ihnen)

(Listen Sie die Top 3 auf, einschließlich Titel und Autor): 
Artikelbezeichnung

Anzahl der Tweets:

(bezogen auf einen Artikel)

Tweets können lediglich in Echtzeit nachvollzogen werden. Aber sie können auch, sofern sie verzeichnet worden sind, auf der jeweiligen Website nachträglich eingesehen werden. Auch können Sie unter Altmetric Bookmarklet www.altmetric.com/bookmarklet.php kostenfrei abgerufen werden.

Tweets oder Blog-Posts über Publikationen

Beratung

Berufspraxis

Gesetze, Regulierung, Richtlinien

Einfluss auf Wirtschaft

Einnahmen

Beratung

Patentzitationen

Patentzitationen

Patentzitationen

Spin-Offs
Artikelbezeichnung

Anzahl der Blog-Posts: (bezogen auf einen Artikel) [Blog-Posts können via Google identifiziert werden. Dazu muss nach dem entsprechenden Publikationsnamen unter Google Blog Search (dazu das Google-Hauptmenü auswählen und die Kategorie „Blogs“ auswählen) recherchiert werden. Dabei können zahlreiche falsche Suchergebnisse ausgeworfen werden, was eine Überprüfung der Ergebnisse und erneute Filterung erforderlich macht.]

Anzahl der Anfragen nach Spezialwissen von außerhalb des akademischen, ökonomischen oder ausbildenden Umfeldes, einschließlich Mitgliedschaft in entsprechenden Komitees:

Beispiele für solche Anfragen/Mitgliedschaften (Benennen Sie die Top 3.).

Beispiele für Einsatz Ihrer Expertise in der Berufspraxis (z. B. Tätigkeit als Anwalt) (Benennen Sie die Top 3.).

Gesetze, Regulierungen, Richtlinien etc., die (zumindest teilweise) basierend auf Ihren Forschungsarbeiten initiiert, entwickelt oder verändert wurden. Bitte kurze Erklärung und Verweise auf Projekte, Publikationen oder andre Belege hierfür (Benennen Sie die Top 3.).

Gesamtes Drittmitteleinkommen (Finanzmittel aus kommerziellen Aktivitäten):

Anzahl Beratungen oder Beratungspositionen für Unternehmen:

Anzahl von Patenten, die Ihre Arbeiten zitieren:

Namen von Patenten, die Ihre Arbeiten zitieren (Benennen Sie die Top 3): [Zitationen von Patenten, sofern welche in Google Scholar Citations aufgeführt sind.]

Anzahl von Zitationen Ihrer Patente (wenn) aus wissenschaftlichen Dokumenten:

[Zitationen von Patenten, sofern welche in Google Scholar Citations aufgeführt sind.]

Anzahl von Zitationen Ihrer Patente (wenn) aus wissenschaftlichen Dokumenten:

[Zitationen von Patenten, sofern welche in Google Scholar Citations aufgeführt sind.]

Anzahl von Ihnen realisierter Spin-Off-Unternehmungen: 


\section{Expertise}

Einfluss auf Lehre
Preise

Online-Views

Syballus-

Erwähnungen

Gastvorlesungen

Datensätze oder Software-Downloads
Lehrpreise (Benennen Sie die Top 3.).

Anzahl der Aufrufe/Views Ihrer Top 3 SlideShare oder Youtube-

Präsentationen, sofern nennenswert.

\begin{tabular}{lll} 
& & Aufrufe/ \\
Präsentations-URL: & Views & \\
\cline { 2 - 3 } Präsentations-URL: & Aufrufe/ & \\
\cline { 2 - 3 } & Views & \\
Präsentations-URL: & Aufrufe/ & \\
\cline { 3 - 3 } & Views &
\end{tabular}

[Sie können an dieser Stelle auch vergleichbare andere Webseiten, wie bspw. Vimeo oder andere Online-Lernplattformen, und die dazugehörige Anzahl an Aufrufen angeben.]

Anzahl der Online-Lehrmaterialien oder Skripte, welche Ihre akademische Arbeit beinhalten (Benennen Sie die Top 3.).

\begin{tabular}{ll} 
Veröffentlichung: & Syllabus \\
\cline { 2 - 2 } Veröffentlichung: & Vermerk: \\
\cline { 2 - 2 } Veröffentlichung: & Syllabus \\
\cline { 2 - 2 } & Vermerk: \\
& Syllabus \\
Vermerk:
\end{tabular}

[Hinweis: Syllabi können via Google identifiziert werden. Dazu nach folgenden Begrifflichkeiten suchen:

Syllabus „[Titel der Publikation]“ oder „Leseliste“ „[ Titel der Publikation]“, in denen [Titel der Publikation] eine Schlüsselpublikation ist. Dazu können Sie auch verschiedene Sprachversionen des Begriffs „reading list“ ausprobieren. Da dies eine sehr zeitintensive Suche ist, benennen Sie an dieser Stelle nur Syllabus-Erwähnungen, die im Rahmen Ihrer Arbeit von sehr großer Bedeutung sind.]

Gesamtverkaufszahlen Ihrer Lehrbuchexemplare:

[Genutzt werden können Amazon-Verkaufsplatzierungen für ähnliche Bücher, wenn Verkaufszahlen nicht vorhanden sind] (Benennen Sie die Top 3.). Um dies zu tun, suchen Sie nach Ihrem Buchtitel oder der ISBN-Nummer in Amazon.com und finden Sie „Amazon Best Sellers Rank“ in den Produktbzw. Artikeldetails

Anzahl von Gastvorlesungen an anderen Universitäten:

Anzahl der Downloads von Datensätzen oder Anwendungen (Benennen Sie die Top 3.).

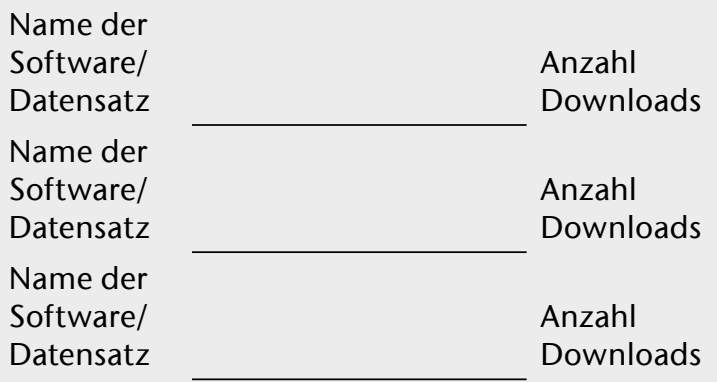

[auch Zitationen aus Google Scholar können hierfür genutzt werden] Andere Einflussarten (Benennen Sie die Top 3 und erläutern diese.). 


\section{EINSATZMÖGLICHKEITEN DES ACUMEN-PORTFOLIOS UND SCHLUSSFOLGERUNGEN}

Die Einsatzmöglichkeiten des ACUMENPortfolios sind vielfältig. So können das Portfolio oder nur Teilelemente aus dem Portfolio genutzt werden, um die Eignung von Wissenschaftlern für offene Positionen an Hochschulen und außeruniversitären Forschungseinrichtungen zu bewerten. Dabei werden systematisch unterschiedliche Aspekte berücksichtigt, die weit über die Publikationsaktivitäten in hochrangigen Zeitschriften oder die Summe eingeworbener Drittmittel hinausgehen. In diesem Zusammenhang kann das Portfolio auch angewandt werden, um zu entscheiden, wann Wissenschaftler in höhere Positionen wechseln sollten. Das Portfolio stellt die Bewertung auf eine transparente Basis und unterstützt eine systematische Karriereplanung und -entwicklung. Auch im Zusammenhang mit der Bewerbung um Drittmittel kann das Portfolio eine geeignete Grundlage darstellen, um die Vergabeentscheidungen von Forschungsmitteln zu unterstützen. Ein besonderer Aspekt liegt in der Möglichkeit der Selbstevaluation und auch im Monitoring der individuellen Entwicklung durch die Wissenschaftler. In diesem Sinne wird das ACUMENPortfolio dann nicht in erster Linie für externe Evaluatoren genutzt, sondern ermöglicht die individuelle Reflektion und zeigt Stärken und Schwächen auf als Grundlage für eine weitere, eigene Karriereplanung.

Mit dem Ansatz der im ACUMEN-Portfolio vorgestellten Indikatoren wird der mit dem Begriff "publish or perish“ assoziierte Bewertungsmaßstab „Anzahl der Publikationen in hochrangigen Journals" zumindest um Indikatoren ergänzt, die eine weitaus differenzierte Bewertung von Wissenschaftlern und ihren Leistungen unter Berücksichtigung des jeweiligen Kontextes erlauben. Im ACUMEN-Portfolio werden auch Aspekte berücksichtigt, die z.B. Aktivitäten der Wissenschaftler im Bereich Technologietransfer oder in der akademischen Lehre erfassen und stärker gewichten als bisher.

\section{LITERATUR}

ACUMEN (2014a) ACUMEN-Periodic Report: Period 2.

ACUMEN (2014b) Guidelines for Good Evaluation Practice with the ACUMEN Portfolio.

Bar-Ilan I (2008) Which h-index? - A comparison of WoS, Scopus and Google Scholar. Scientometrics 74(2):257-271. doi: 10.1007/s11192-008-0216-y

Bar-Ilan J, Hartmann F, Havemann F, Larsen B, Mietzer D, Scharnhorst A, Tatum CC (2014) Are You Being Evaluated? Need for New Approaches in Evaluation Practices. In: Kindling M, Greifeneder E (eds) iConference 2014 Proceedings. IDEALS, Urbana, IL, ISBN: 978-0-98849001-7, pp 1204-1206. doi: 10.9776/14215

Hirsch JE (2005) An index to quantify an individual's scientific research output. Proc Natl Acad Sci USA 102(46):16569-16572. doi: 10.1073/pnas.0507655102

Kousha K, Thelwall M (2012) Motivations for Citing YouTube Videos in the Academic Publications: A Contextual Analysis. In: 17th International Conference on Science and Technology Indicators (STI), 5-8 Sep 2012, Montréal, Canada

Kousha K, Thelwall M, Abdoli M (2012) The role of online videos in research communication: A content analysis of YouTube videos cited in academic publications. J Am Soc Inf Sci Tec 63(9):1710-1727. doi: 10.1002/asi.22717

Kousha K, Thelwall M (2013) Evaluating the Web Research Dissemination of EU Academics: A MultiDiscipline Outlink Analysis of Online CVs. In: 14th International Society of Scientometrics and Informetrics Conference, 15-20 Jul 2013, Vienna, Austria

Kousha K, Thelwall M (2014a) An automatic method for extracting citations from Google Books. J Assn Inf Sci Tec (Online Version before inclusion in an issue). doi $10.1002 /$ asi. 23170

Kousha K, Thelwall M (2014b) Web Impact Metrics for Research Assessment. In: Cronin B, Sugimoto CR (eds) Beyond Bibliometrics. Harnessing Multidimensional Indicators of Scholarly Impact. MIT Press, Cambridge MA, ISBN: 978-0-262-02679-6, pp 289-306

Más-Bleda A, Aguillo IF (2013) Can a personal website be useful as an information source to assess individual scientists? The case of European highly cited researchers. Scientometrics 96(1):51-67. doi: 10.1007/ s11192-013-0952-5

Tatum C, Wouters P (2013) Acumen Portfolio: Resources for Evaluation of Individual Researchers. In: euroCRIS Membership Meeting, 14 Nov 2013, Porto, Portugal

Thelwall M, Kousha K (2014a) Academia.edu: Social network or Academic Network? J Assn Inf Sci Tec 65(4):721-731. doi: 10.1002/asi.23038

Thelwall M, Kousha K (2014b) ResearchGate: Disseminating, communicating, and measuring Scholar-ship? I Assn Inf Sci Tec (Online Version before inclusion in an issue). doi: 10.1002/asi.23236

Wildgaard L (2014) Just Pimping the CV? The Feasibility of Ready-to-use Bibliometric Indicators to Enrich Curriculum Vitae. In: Kindling M, Greifeneder E (eds) iConference 2014 Proceedings. IDE-ALS, Urbana, IL, ISBN: 978 0-9884900-1-7, pp 954-958. doi: 10.9776/14326

\section{AUTOREN}

Prof. Dr. rer. pol. Dana Mietzner Technische Hochschule Wildau Forschungsgruppe Innovations- und Regionalforschung dana.mietzner@th-wildau.de

Dr. phil. Frank Hartmann

Technische Hochschule Wildau

Forschungsgruppe Innovations- und Regionalforschung frank.hartmann@th-wildau.de 